\title{
A Total Sample Preparation Method for the Measurement of Airborne Asbestos and Other Fibers by Optical and Electron Microscopy
}

\author{
Norihiko KOHYAMA and Shizue KURIMORI
}

National Institute of Industrial Health, 21-1,

Nagao 6-chome, Tama-ku, Kawasaki 214, Japan

(Received March 26, 1996 and in revised form April 24, 1996)

\begin{abstract}
A total sample preparation method utilizing one membrane filter for all or some of the measurements by phase contrast optical microscopy (PCM), optical microscopy (OM), dispersion staining polarized microscopy (DS/PLM), scanning electron microscopy (SEM), and transmission electron microscopy (TEM), was developed in order to evaluate airborne asbestos and other mineral fibers comprehensively, including fiber number, sizes and types, in various environments. This method consists of two alternate procedures: parallel and serial preparation. The former uses different portions of a filter for each measurement of PCM, OM, DS/PLM, SEM and TEM. The latter uses a single filter portion for all OM, SEM and TEM measurements by which the same area and same fibers on the filter can be observed. This allows fiber numbers to be directly compared using these measurements on the same filter area, and the fibers observed by $O M$ can be also examined by SEM and/or TEM equipped with an energy dispersive X-ray spectrometer (EDX) to determine the types and precise sizes. Using this method, it was found that $O M$ measurements developed in this study can visualize thinner fibers than PCM measurements and make it easier to count fibers. We also found that currently used PCM measurement can detect fibers thicker than about 0.4-0.5 $\mu \mathrm{m}$ in diameter. This total sample preparation method can be used not only for air samples, but also for liquid and biological tissue samples.
\end{abstract}

Key Words: Asbestos - Fibrous Minerals - Airborne Fibers - Fiber counting - Air sampling - Membrane Filter - Optical Microscopy - Electron Microscopy

\section{INTRODUCTION}

Airborne asbestos fibers in working environments, where the source and type of asbestos fibers are known, are usually analyzed by phase contrast optical microscopy (PCM) in many countries with legal regulations, ${ }^{1,2}$. PCM has many advantages as a conventional analysis method: it is simpler than electron microscopic measurement, it has a long history of use in occupational environments, and 
risk assessment of asbestos-exposed workers has been done on the basis of the data measured by PCM. However, PCM has some drawbacks; for example, it is not specific to asbestos, it cannot distinguish asbestos fibers from other mineral fibers, and it cannot detect thin fibers but rather only comparatively large airborne fibers. Recently, the needs of asbestos measurement have been greatly changing; that is, environments to be monitored have diversified from work sites to general ambient air ${ }^{3)}$, and asbestos substitutes are actively used in industries, which means fiber types in working environment are changing, and knowledge regarding fiber carcinogenicity (dependent on fiber size, durability in the human body, etc.) is accumulated. It is widely believed that fiber type and size are both important factors in the adverse health effects of fibrous minerals, ${ }^{4}$. These recent changes concerning fibrous minerals including asbestos require comprehensive analysis of airborne fibers in various environments, especially determination of fiber types and size distributions, including very thin fibers. Using PCM, however, it is difficult to identify fiber type and to quantify fiber size, especially diameter, because of limited resolution.

To compensate for these limitations, electron microscopy (EM) including scanning electron microscopy (SEM) and transmission electron microscopy (TEM) has gradually been used in industrial hygiene fields ${ }^{6-9}$. Most of these EM measurements, however, have been done independently from PCM measurement, or use different filter samples from those used in PCM measurement. Until now, it has been almost impossible for analysts to observe the same area of a filter with EM that had also been observed by PCM. Therefore, our goal was to develop a new sample preparation method for measurement by PCM, OM, SEM and TEM using the same filter sample with collected airborne dust. This method has made it possible for analysts to directly compare the results obtained by these different tools on the same filter area and fibers.

\section{SAMPLE PREPARATION METHODS}

The schema of the total sample preparation method is shown in Figure 1. Asbestos fibers are collected on a mixed cellulose ester membrane (CE) filter with a diameter of either 47 or $25 \mathrm{~mm}$ and a pore size of $0.8 \mu \mathrm{m}$ using an air sampler in various environments. The total sample preparation method consists of two alternate procedures: parallel preparation and serial preparation. The parallel preparation method uses different portions of the same filter sample for measurements of PCM, optical microscopy (OM), dispersion staining polarized microscopy (DS/PLM), SEM and TEM. The serial preparation uses the same single filter portion for all measurements of OM, SEM and TEM (Fig. 2). After the first measurement of OM, the same sample is also observed by SEM, and successively re-treated for TEM, which are designated as $\operatorname{SEM}(2)$ and $\operatorname{TEM}(1)$, respectively. 
a) Parallel preparation

b) Serial preparation

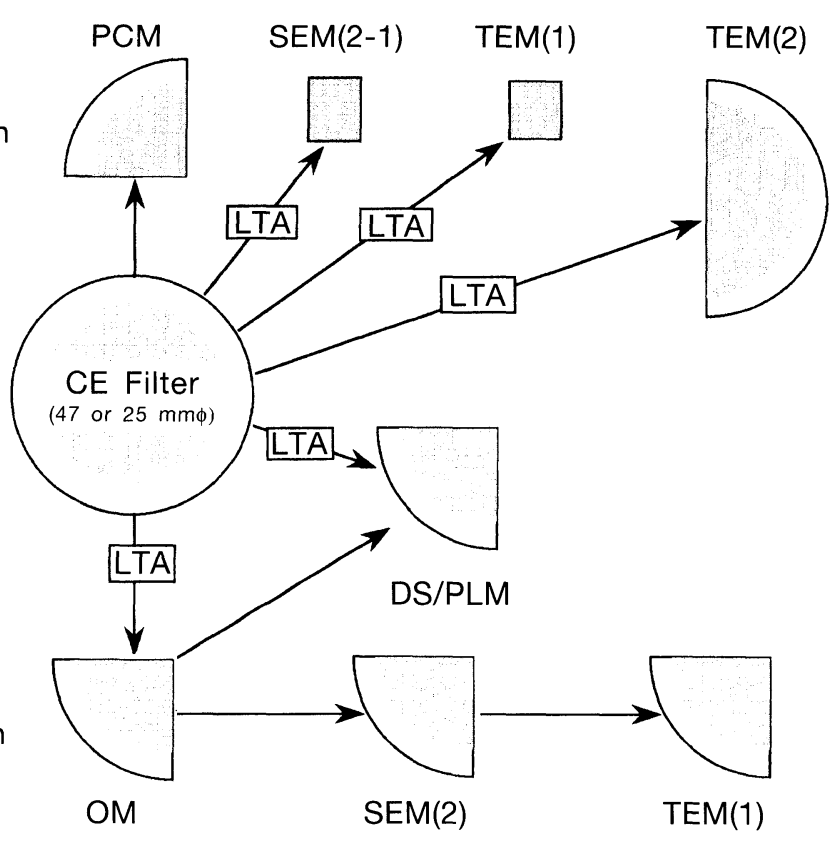

Fig. 1. Schema of total sample preparation method for measurement of airborne asbestos and other fibers using one membrane filter.

Capital letters: indicate sample preparation and analytical method (see text), LTA: low-temperature ashing.

Of course, when the analyst needs only SEM or TEM measurement, he can directly proceed to those preparations as shown in the parallel preparation in Figure 1. The advantage of the sample preparation methods (except $\operatorname{TEM}(2)$ ) is that the same state on the initial filter can be observed; this is the so-called "direct-transfer method". In the TEM(2) measurements, the initial dust state on the filter was changed in the final TEM sample; this is called the "indirect-transfer method", and the analyst can adjust the dust concentration (to concentrate or dilute) on the final TEM specimen through this sample transfer procedure.

1) PCM: This traditional method is already utilized in many countries ${ }^{1,2)}$. A portion of the CE filter sampled airborne dust is attached to a glass slide using acetone vapor, then a drop of triacetin solution is placed on it and covered with a cover slip. This method is detailed in the references ${ }^{1,2)}$.

2) OM: Another portion of the CE filter is attached to a glass slide using acetone vapor, so that the dust side of the filter faces the glass slide. Then lowtemperature ashing is done in an oxygen plasma asher at 280-300 watts for 30-60 


\section{b) Serial Preparation}

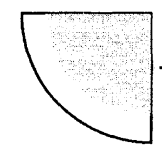

a quarter of filte

slide using hot

acetone vapor

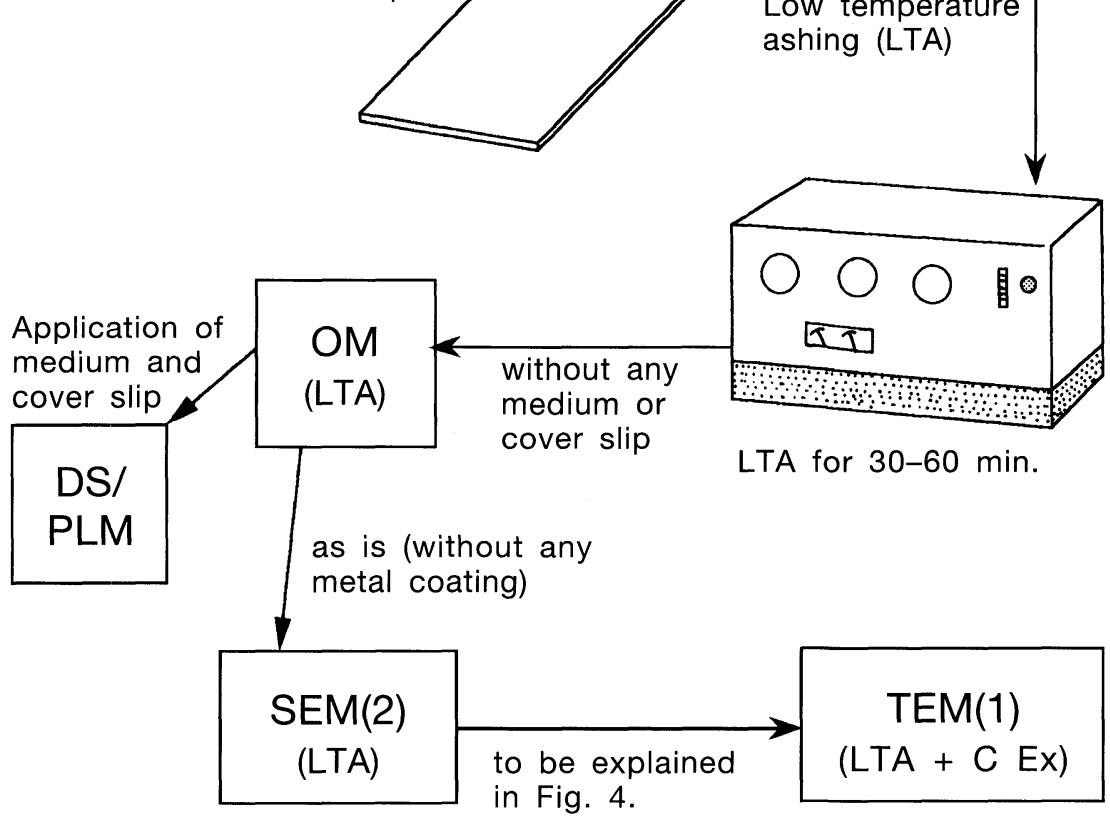

Fig. 2. Schema of serial preparation in the total sample preparation method.

minutes. By this ashing, the filter and organic materials are burnt away and inorganic dust including asbestos and other mineral fibers remained on the glass slide. The fibers on the glass slide are counted by $\mathrm{OM}$ in a limited area on the slide without any application of medium and cover slip. The fiber counting follows the same counting rules as the PCM method. In this measurement, it is important that the objective aperture of $\mathrm{OM}$ is stopped down to be able to visualize fine fibers. Of course, PCM can be used for this slide sample, but the use of OM is desirable because it gives better contrast and sharper images.

3) DS/PLM: DS/PLM has been widely used for identification of raw fibrous materials for asbestos abatement requirements for asbestos sprayed in buildings ${ }^{10)}$. However, DS/PLM measurement has not been applied to airborne fibers because of the interference of the sampling filter itself. The authors tested whether the glass slide sample after low temperature ashing could be used for measurement 
of DS/PLM. Applying a medium solution of a proper index to the glass slide, relatively large airborne fibers could be observed. This DS/PLM measurement can be also applied to the same filter sample used in OM observation to identify the types of relatively large fibers. As a medium solution is applied on the glass slide, the sample cannot be used for the further examination by SEM and TEM (Fig. $1)$.

4) SEM(2): After the OM measurement, the glass slide sample is set directly in the SEM instrument to observe the fibers. Without any metal coating such as gold or carbon to enhance conductivity, fibers on the glass slide can be seen by a conventional SEM. However, if the analyst uses a low-vacuum SEM, which has been recently developed and sold under the name of Environmental SEM ${ }^{\circledast}$, Natural SEM ${ }^{\circledR}$, Wet $S E M^{\circledR}$ and etc, he can observe clearer images of the fibers without any metal coating. Using a low-vacuum SEM, fibers equivalent to those observed by OM (longer than $5 \mu \mathrm{m}$ and with a minimum detectable fiber diameter of about $0.25 \mu \mathrm{m})$, can be observed at magnifications of about 2,000 or $4,000 \mathrm{x}$ on the CRT screen directly (visually). All the fibers corresponding with the above ranges are counted at this magnification for a certain area. Energy dispersive X-ray (EDX) analysis can be done for each fiber when the analyst uses an analytical SEM, but he must pay attention to contaminant $\mathrm{X}$-rays from the base of glass slide, such as $\mathrm{Si}, \mathrm{Al}, \mathrm{Ca}, \mathrm{Na}$, etc.

5) $\operatorname{SEM(2-1):~When~the~analyst~does~not~intend~to~conduct~further~measurement~}$ by TEM, as in the case of parallel preparation, the following sample preparation can be used. A piece of the CE filter is attached to a small nickel plate (about $1 \mathrm{~cm}^{2}$ ) using acetone vapor, and then ashed in a oxygen plasma asher at 280-300 watts for 30-60 minutes. After carbon evaporation coating is applied on the surface, it is mounted on an SEM specimen disk using conductive carbon adhesive tape and carbon paste to enhance the electron conductivity. Fibers on this specimen are clearly observed by a conventional SEM instrument and their chemical composition can be analyzed by EDX analysis without any interference of characteristic X-ray peaks from the base plate, with the exception of nickel X-ray peaks. The technical flow chart for this preparation is shown in Figure 3.

6) $\operatorname{TEM}(1)$ : After the $O M$ and/or the $\operatorname{SEM(2)~measurements,~the~glass~slide~}$ sample is used in the TEM(1) method, which is a "direct-transfer method", in serial preparation. The flow chart for this procedure is illustrated in Figure 4. The sample area on the slide is marginated ("corner-affixed") with cellophane adhesive tapes (Cellotape ${ }^{\circledast}$, Nichiban Co., Japan) and covered with $8-9 \%$ of polyvinyl alcohol (PVA) solution using a pipette. The volume is about 1-2 $\mathrm{ml}$ for about 2-3 $\mathrm{cm}^{2}$. The PVA solution on the slide is dried for a few hours at about $50^{\circ} \mathrm{C}$ 


\section{$\operatorname{SEM}(2-1)$}

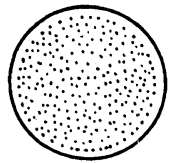

Sampling with $C E$ filter $(47 \mathrm{~mm} \phi$, $0.8 \mu \mathrm{m}$ pore size)

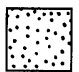

Cut out a portion of the filter

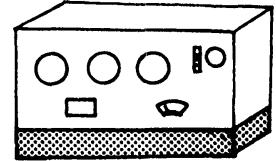

Low Temp. Ashing for 30 $\min$.

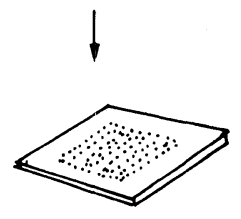

Carbon coating
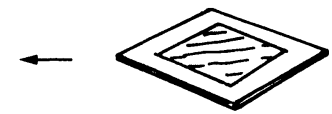

Attach the filter with the dust side facing the Ni-plate or glass slide

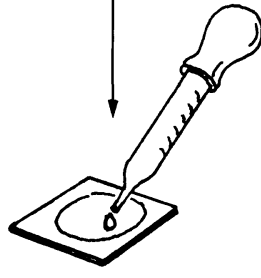

Drop a small amount of acetone on a $\mathrm{Ni}$ plate or glass slide

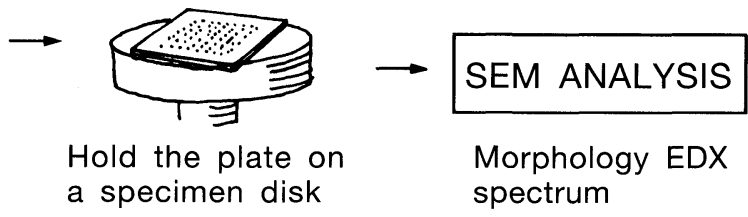

Fig. 3. Flow chart of the sample preparation method for SEM(2-1).

(must not exceed $60^{\circ} \mathrm{C}$ ) or overnight at room temperature. The dried PVA film is removed and turned over (dust side up) and fixed again on the glass slide with new cellophane tapes. After application of relatively heavy carbon evaporation (about 30-50 nm in thickness) on the surface of the PVA film, lattice streaks are made at 2-3 $\mathrm{mm}$ intervals on the carbon layer using a sharp knife. The whole film is floated on hot water in a beaker $(500 \mathrm{ml})$ for several hours to dissolve the PVA film. The beaker should be slightly heated in the mantle-heater, maintaining the high temperature at about $80^{\circ}$ to $70^{\circ} \mathrm{C}$. Small pieces of floating carbon film (2-3 mm square), holding mineral particles, are picked up by 100-200 mesh nickel TEM grids. Generally, 5 to 10 specimen grids are produced.

7) TEM(2): Half of the CE filter can be used for the TEM(2) method, which is an "indirect-transfer method", in parallel preparation"1). This procedure is illustrated in Figure 5. A half of the filter is attached to a clear glass slide using acetone vapor with the dust side facing the glass slide and ashed in a low-tem- 


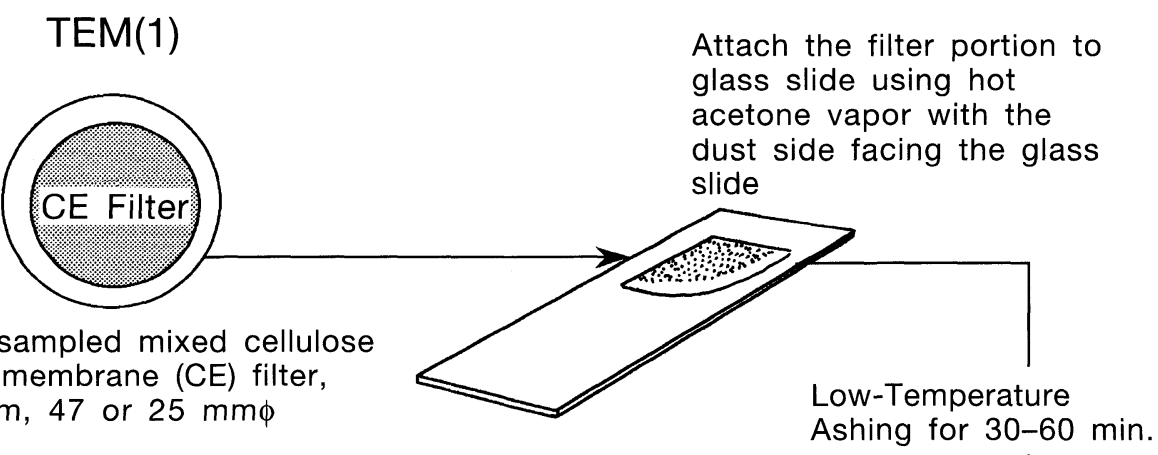

Dust sampled mixed cellulose ester membrane (CE) filter, $0.8 \mu \mathrm{m}, 47$ or $25 \mathrm{mm \phi}$

Ashing for 30-60 min.

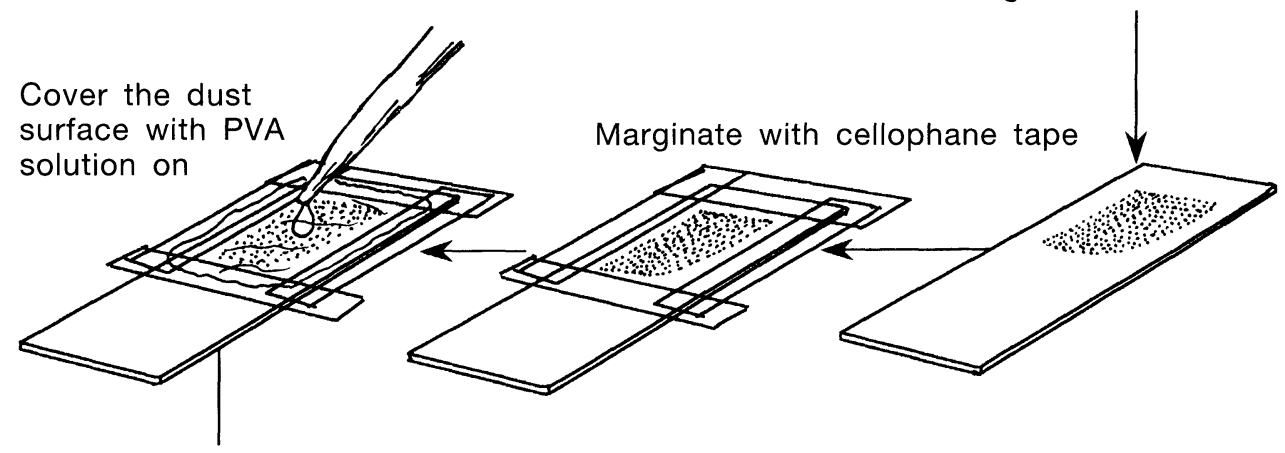

Dry for a few hours at $50^{\circ} \mathrm{C}$, remove the film and turn over, \& fix again on the glass slide with cellophane tape

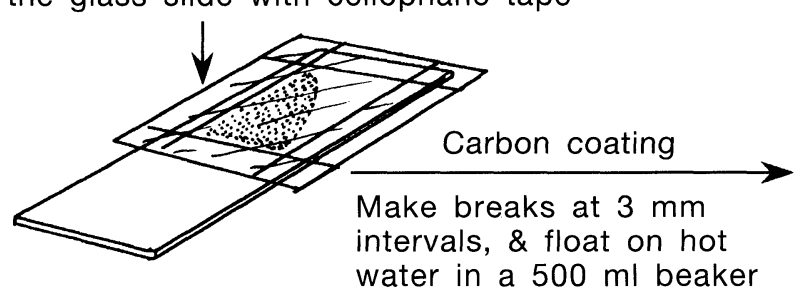

Maintain temperature over $70^{\circ} \mathrm{C}$ for $3-5$ hours water in a $500 \mathrm{ml}$ beaker

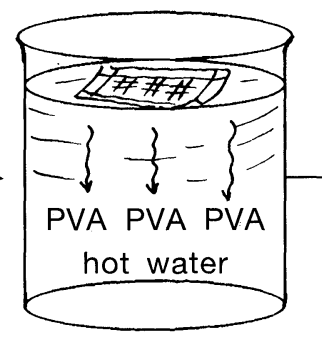

Scoop up the carbon film portions by $\mathrm{Ni}$ TEM-grids

\section{ATEM Analysis}

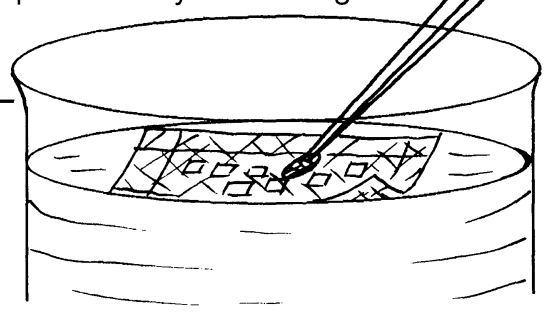

Fig. 4. Flow chart of the sample preparation method for TEM(1). 
$\operatorname{TEM}(2)$

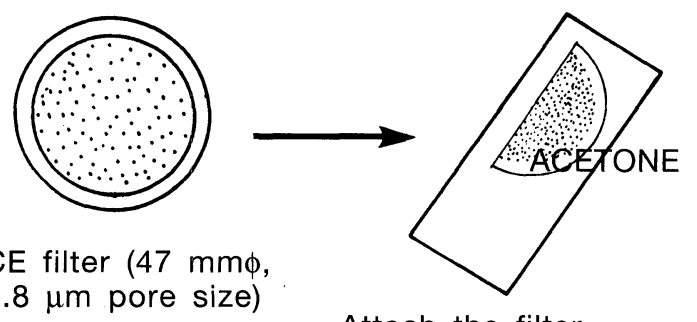

Attach the filter

portion to glass slide

with acetone vapor

\section{PARTICLES}
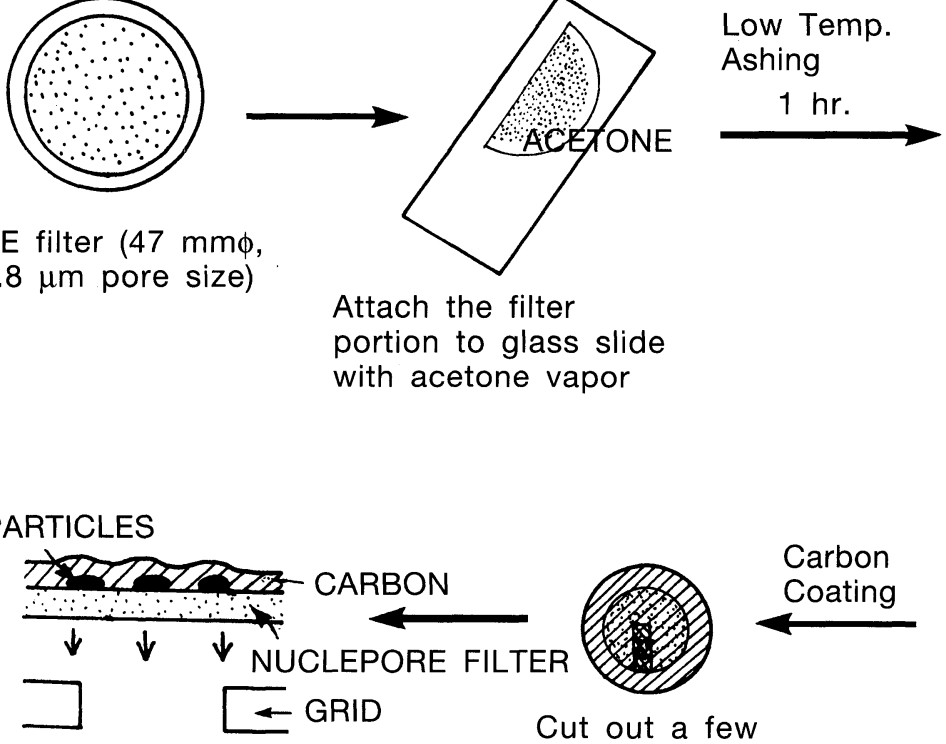

Disperse the dust into isopropanol

$0.8 \mu \mathrm{m}$ pore size)

Cut out a few pieces of filter

(2-3 $\mathrm{mm}$ square)

Disolve the

filter with chloroform

PARTICLE

$\checkmark$
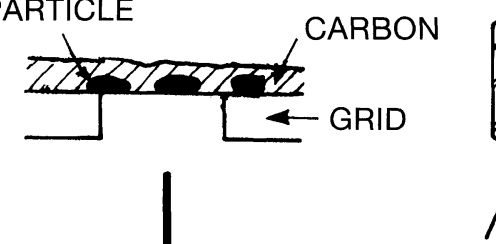

STAINLESS

MESH
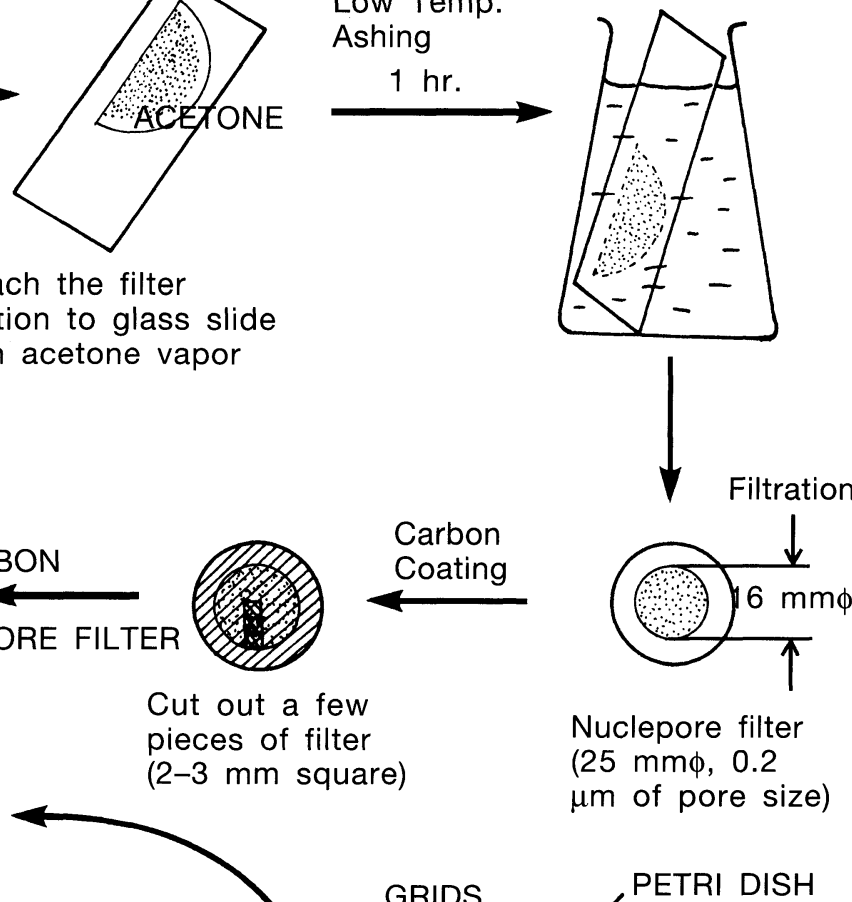

Nuclepore filter

(25 mm $\phi, 0.2$

$\mu \mathrm{m}$ of pore size)
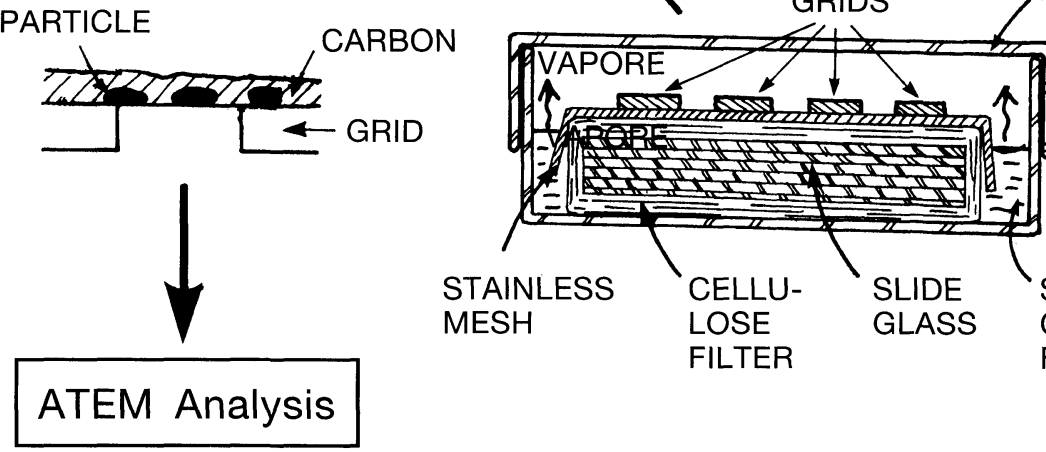

PETRI DISH

LOSE

FILTER

GLASS

SOLVENT

CHLORO-

FORM

Fig. 5. Flow chart of the sample preparation method for TEM(2).

perature plasma asher for about one hour. To suspend the residual mineral dust in isopropanol, the surface of the glass slide is wetted with a few droplets of isopropanol and shaved with a new and oil-removed blade. Both the glass slide and the blade holding the residual dust are then immersed in about $50 \mathrm{ml}$ isopropanol (which should be filtrated by CE filter with a pore size of $0.2 \mu \mathrm{m}$ 
before use) in a 100-ml conical flask, and the dust particles are dispersed by ultrasonification for a short time (about 30 seconds). The suspension is filtrated through a polycarbonate (PC) filter with a pore size of $0.2 \mu \mathrm{m}$ and an effective area of $2.0 \mathrm{~cm}^{2}$. When a CE filter of $47 \mathrm{~mm}$ in diameter (the effective diameter is $40 \mathrm{~mm}$ ) is used, because the effective filtered area is about $12.6 \mathrm{~cm}^{2}$, the dust sample on the half of the CE filter is condensed on the PC filter by about three times through this preparation. If the analyst wants to dilute the initial dust concentration, he may use a smaller portion of the initial filter than the effective area of the next PC filter. The PC filter is then vacuum-coated with a relatively heavy carbon evaporation and a few pieces of the filter (2-3 mm square) are cut out. These small filter pieces are placed on 100-200 mesh nickel TEM grids in a petri dish with chloroform (Jaffe washer) and left overnight or for a few days to dissolve the PC filter. For more rapid and better dissolution of the filter, a condensation washer, consisting of a flask, condenser and cold finger assembly with the mantle-heater, is effective ${ }^{9)}$. Maintaining the dust on the carbon film, the TEM specimen grid is completed.

\section{RESULTS}

Comparison of the data measured by PCM, OM and TEM methods on the samefilter sample

Recently it has been widely recognized that the carcinogenicity of asbestos is strongly related to fiber sizes. Therefore, it is important to know the size distribution of fibers in addition to concentrations in working environments. These results will help explain the actual dose-response relationship of asbestos. Using the total sample preparation method, the concentration and size distribution of airborne asbestos in an asbestos factory were measured by the PCM, OM, and TEM methods. The factory had been making asbestos cement boards using chrysotile and amosite, but stopped using amosite in 1994. Air samples of about 300 liters were collected in 1992 on CE filters of $25 \mathrm{~mm}$ in diameter at the work sites where opening asbestos bags and milling occurred. The sample preparations for each instrument followed the methods mentioned above.

1) Clearness of images: The photographs taken by PCM and OM are shown in Figure 6 (chrysotile) and Figure 7 (amosite). It is obvious that the fibers observed by $\mathrm{OM}$ are clear and sharp compared with those observed by PCM and the OM method can produce precise data for fiber counting. Chrysotile fibers observed by the measurements of the PCM, OM and TEM(1) method are shown in Figure 8 , compared at the same magnification. The sharpness of the images is, in the descending order, TEM, OM and PCM. The photograph by the TEM(1) method in Figure 8 can be further enlarged, as shown in Figure 9. We can also see from 


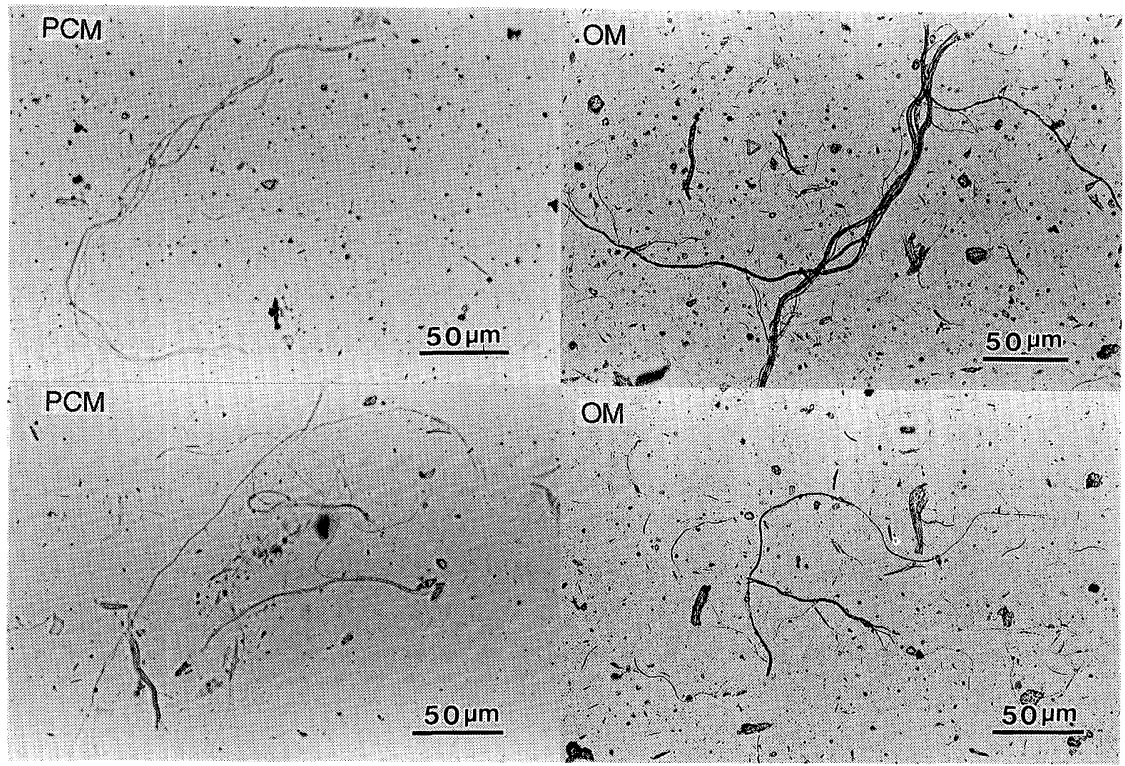

Bar: $50 \mu \mathrm{m}$

Fig. 6. Comparison of the photographs taken by PCM and OM for airborne chrysotile fibers in an asbestos factory.

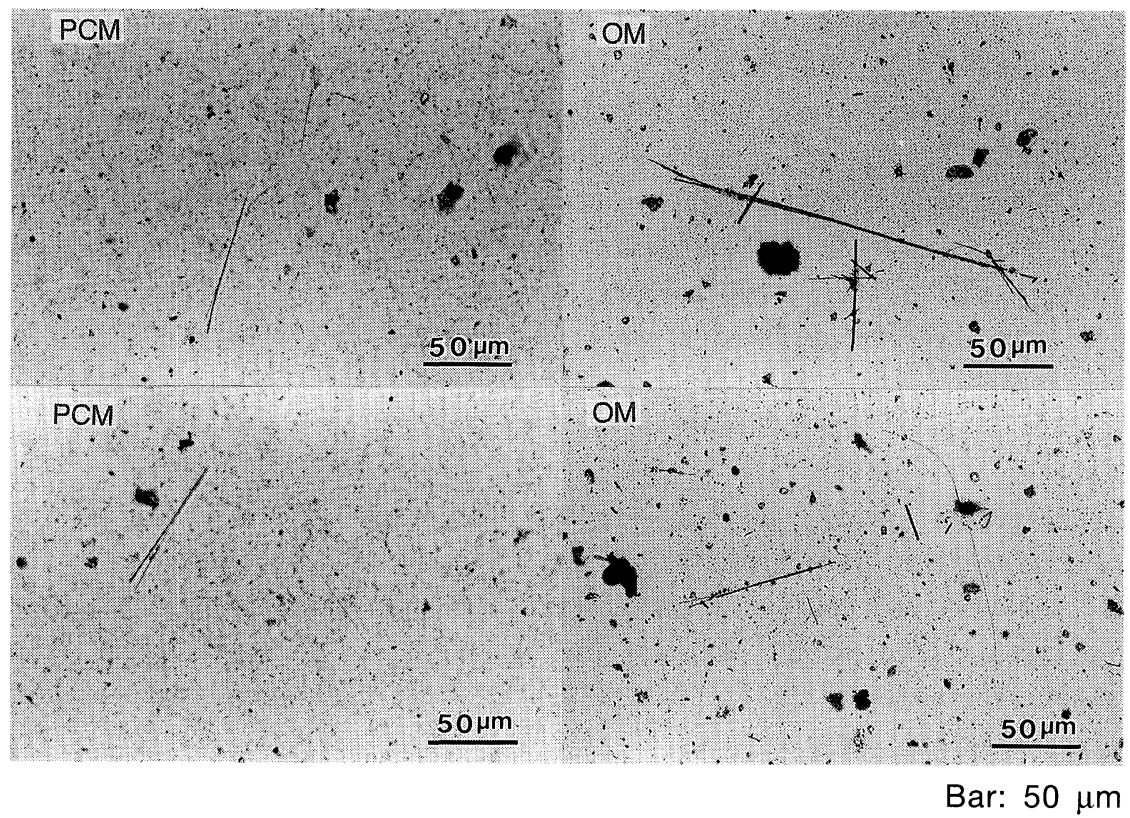

Fig. 7. Comparison of the photographs taken by PCM and OM for airborne amosite fibers in an asbestos factory. 


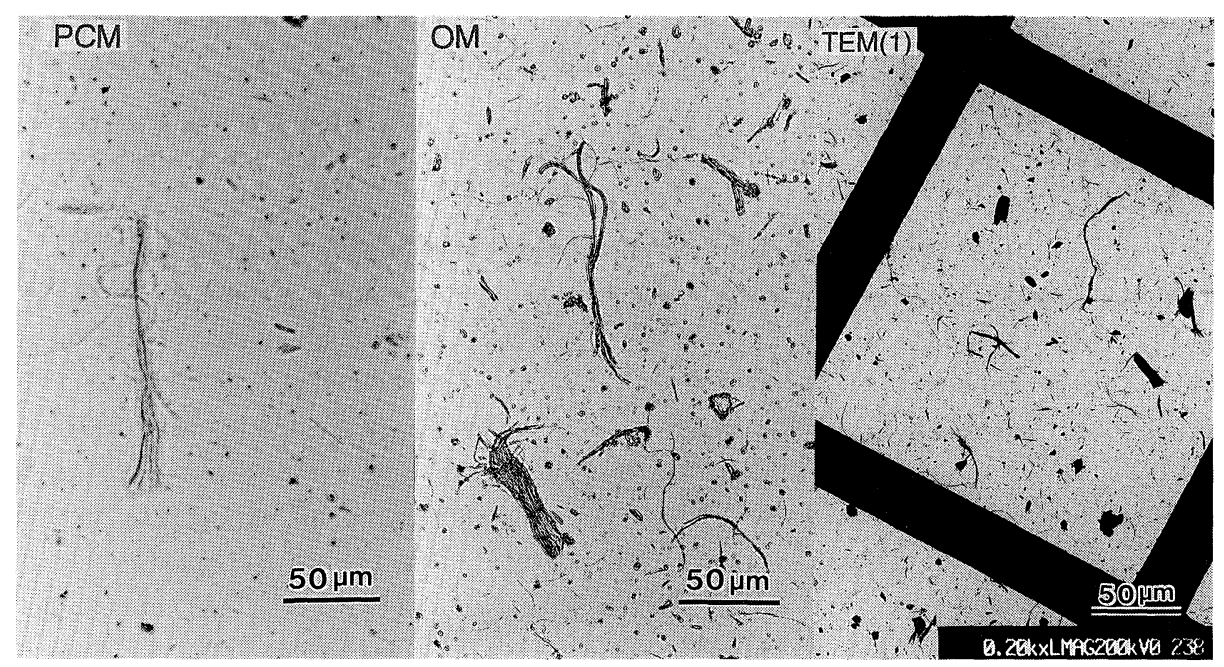

Fig. 8. Comparison of the photographs taken by PCM, OM and TEM(1) at the same magnification for airborne chrysotile fibers in an asbestos factory.

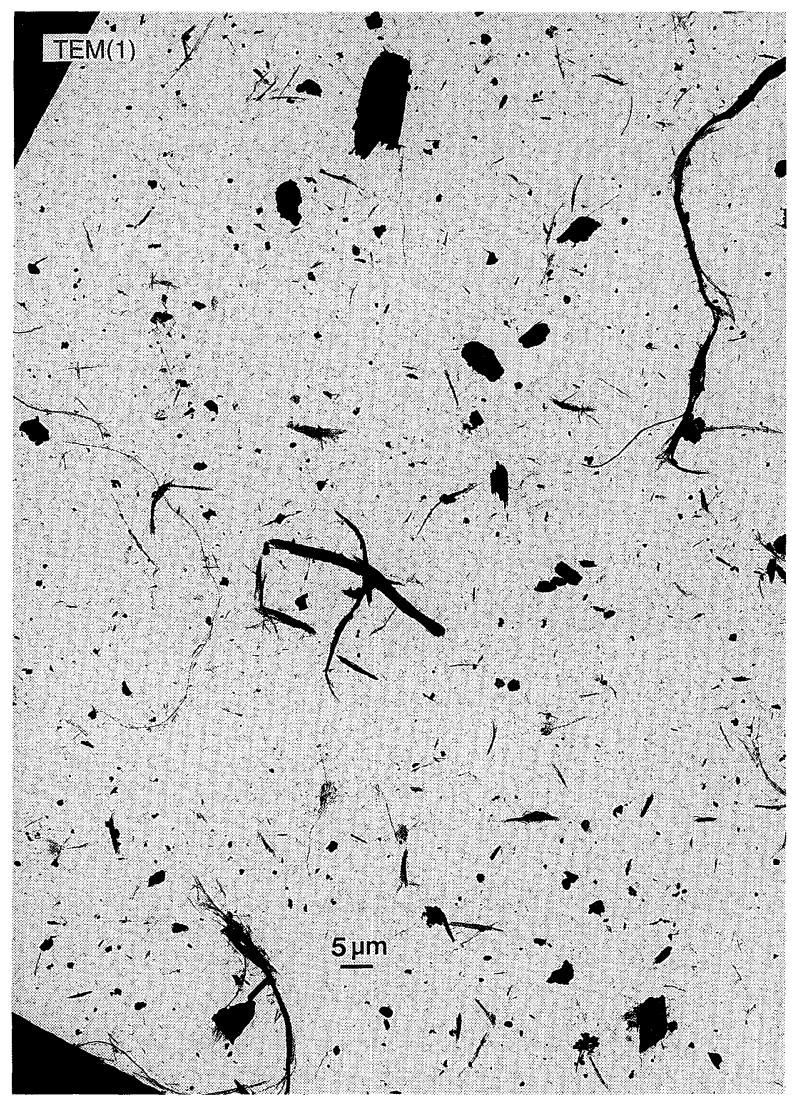

Fig. 9. The photograph of airborne chrysotile fibers taken by $\operatorname{TEM}(1)$, a further enlargement the same TEM(1) photo in Fig. 8. 
Figure 9 that the TEM(1) method can visualize almost all fibers down to $0.02 \mu \mathrm{m}$ in diameter. Generally, as a TEM photograph can be enlarged to a far larger size, we can measure the complete fiber size distribution precisely on the enlarged TEM photograph.

2) The fiber concentration and size distribution of airborne asbestos: The asbestos fiber concentrations were obtained by the measurements using the PCM, OM and TEM(1) methods (Table 1). As a reference, the data measured by PCM for filter samples treated only with acetone vapor but not triacetin are shown as PCM (*) in Table 1. Fiber counting by PCM and OM were done at the magnification of $400 \mathrm{x}$. All fibers longer than $0.5 \mu \mathrm{m}$ in length and wider than $0.02 \mu \mathrm{m}$ in diameter were counted by the $\operatorname{TEM}(1)$ measurement, and as examples, the size distribution data for samples 1 and $\mathrm{A}$ in Table 1 are shown in Figures 10 and 11. Asbestos types on the filter were chrysotile for samples 1-5 and mostly amosite for samples A-E in Table 1. The TEM data in Table 1 are the concentrations calculated for only fibers longer than $5 \mu \mathrm{m}$ and wider than $0.2 \mu \mathrm{m}$ in diameter (the PCM-equivalent fibers ${ }^{6}$ ) within all the observed fibers. The fiber concentrations obtained by OM were about two times higher than those of PCM. The concentration data by TEM(1) were also higher than those of PCM by about 2-4 times, and a little bit higher than those of OM.

When the concentrations of chrysotile and amosite fibers were compared between data for PCM and TEM(1), the concentrations of chrysotile fibers obtained by TEM(1) were 3-4 times (3.87 on average) higher than those obtained by PCM,

Table 1. Airborne asbestos concentrations in an asbestos factory measured by PCM, OM and TEM.

\begin{tabular}{cccccccc}
\hline & \multicolumn{3}{c}{ Fiber Concentration (f/ml) } & \multicolumn{4}{c}{ Ratio of Conc. by Two Different Methods } \\
Sample & PCM $\left(^{*}\right)$ & PCM & OM & TEM(1) & OM/PCM & TEM(1)/PCM & TEM(1)/OM \\
\hline 1 & 0.22 & 0.67 & 1.25 & 2.09 & 1.87 & 3.12 & 1.67 \\
3 & 0.17 & 0.51 & 1.17 & 2.35 & 2.29 & 4.61 & 2.01 \\
4 & 0.18 & 0.32 & 1.03 & 1.24 & 3.22 & 3.88 & 1.20 \\
5 & 0.11 & 0.45 & 0.98 & 1.56 & 2.18 & 3.47 & 1.59 \\
\cline { 5 - 7 } & & & & & $2.39(0.50)$ & $3.87(0.61)$ & $1.62(0.29)$ \\
& & & & & & & 2.73 \\
A & 0.07 & 0.11 & 0.24 & 0.30 & 2.18 & 2.17 & 1.25 \\
B & 0.09 & 0.12 & 0.21 & 0.26 & 1.75 & 2.15 & 1.08 \\
D & 0.16 & 0.13 & 0.26 & 0.28 & 2.00 & 1.61 & 1.26 \\
E & 0.16 & 0.18 & 0.23 & 0.29 & 1.28 & $2.17(0.40)$ & $1.21(0.07)$ \\
\hline
\end{tabular}

1-5: chrysotile only, A-E: mostly amosite, $(*)$ : treated acetone only, without triacetine, TEM(1): counted asbestos fibers greated than $5 \mu \mathrm{m}$ in length and $0.2 \mu \mathrm{m}$ in diameter, ( ): standard deviation. 

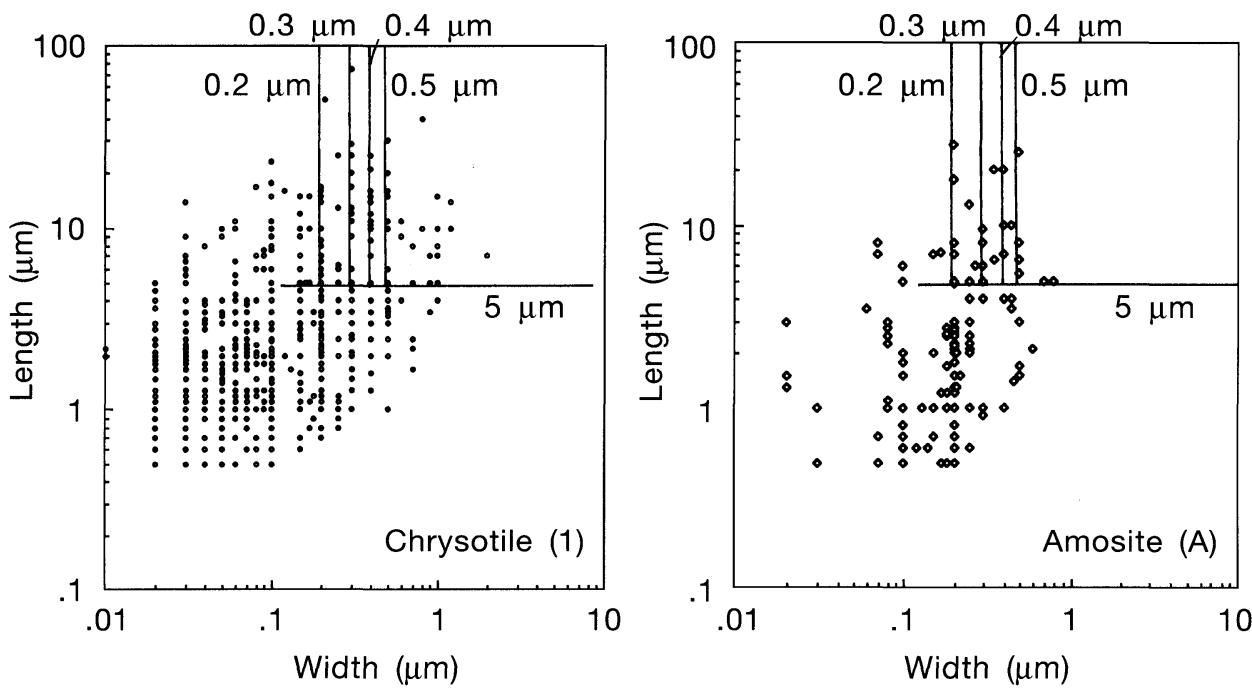

Fig. 10. Scatter graphs of the size distributions of airborne chrysotile and amosite fibers in an asbestos factory, as measured by $\operatorname{TEM}(1)$.

Chrysotile (1): sample 1 in Table 1, Amosite (A): sample A in Table 1, The fibers greater than the indicated sizes in this figures were used for the calculation of the concentrations shown in Table 2 .
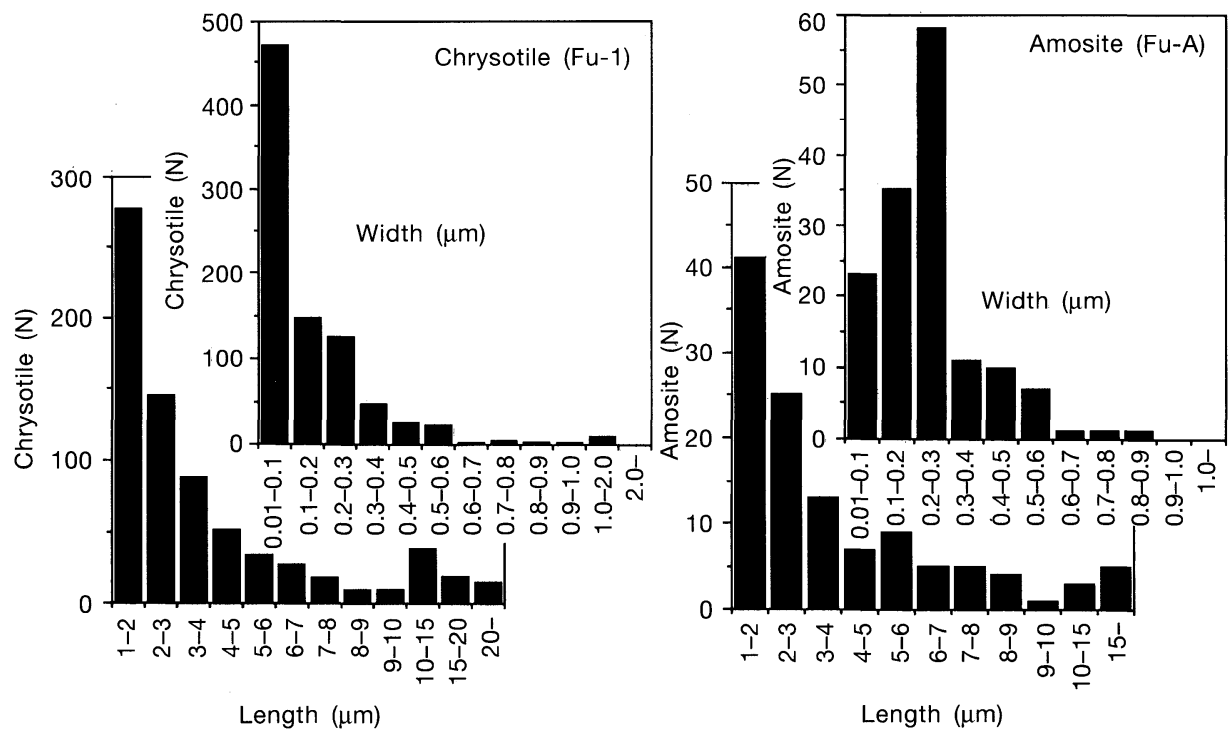

Fig. 11. Comparison of the size distributions of airborne chrysotile and amosite fibers in an asbestos factory measured by $\operatorname{TEM}(1)$. 
whereas the concentrations of amosite were 2-3 times (2.17 on average) higher, as indicated in Table 1. This means that using PCM, it is slightly more difficult to see chrysotile fibers than amosite fibers. However, when the ratios of TEM/ OM were compared for each asbestos type, they were not so different, as shown in Table 1; this means that OM can visualize well for the both kinds of fibers.

3) The minimum fiber diameters detectable by PCM: As shown in Figures 10 and 11 , the diameter of chrysotile fibers were mostly thinner than those of amosite fibers. Amosite fibers had diameters on the order of $0.2-0.3 \mu \mathrm{m}$, while chrysotile fibers measured $0.01-0.1 \mu \mathrm{m}$. When the asbestos concentrations were calculated for all these fibers, chrysotile concentration was $11.51 \mathrm{f} / \mathrm{ml}$, and that of amosite was $1.24 \mathrm{f} / \mathrm{ml}$. These concentrations were about 18 and 11 times of those measured by PCM, respectively; in other words, only $6 \%$ of chrysotile fibers and $9 \%$ of amosite fibers were counted by PCM. Table 2 shows the fiber concentrations calculated by fiber diameter, within all the fibers measured by TEM. From this data, it was estimated that fibers larger than about $0.5 \mu \mathrm{m}$ in diameter can be detected by the current PCM method. In our previous data ${ }^{12)}$, the detection limit of PCM was estimated to be about $0.4 \mu \mathrm{m}$. The OM method can visualize thinner fibers than can be seen by the PCM method at the same magnification, as shown in Table 1.

4) SEM observation for uncoated samples: The SEM photographs in Figure 12 were obtained by a low-vacuum SEM without any metal evaporation coating, showing airborne amosite fibers in work site B in Table 1. It is clearly shown that satisfactorily clear images of fibers can be obtained by a low-vacuum SEM for samples without metal coating, so that the $\operatorname{SEM}(2)$ specimen can be used for the TEM(1) preparation method for TEM observation.

Table 2. Fiber concentrations of chrysotile and amosite calculated by fiber diameter category.

\begin{tabular}{cccc}
\hline & Diameter $(\mu \mathrm{m})$ & Chrysotile $(\mathrm{f} / \mathrm{ml})$ & Amosite $(\mathrm{f} / \mathrm{ml})$ \\
\hline \multirow{2}{*}{ TEM } & $>0.2$ & $2.09 *$ & $0.30^{*}$ \\
& $>0.3$ & 1.51 & 0.23 \\
& $>0.4$ & 1.15 & 0.19 \\
& $>0.5$ & 0.77 & 0.12 \\
\hline PCM & & $0.67 *$ & $0.11^{*}$ \\
\hline
\end{tabular}

*: these data correspond to samples 1 and $\mathrm{A}$ in Table 1; Fiber lengths calculated here are equal or longer than $5 \mu \mathrm{m}$. 

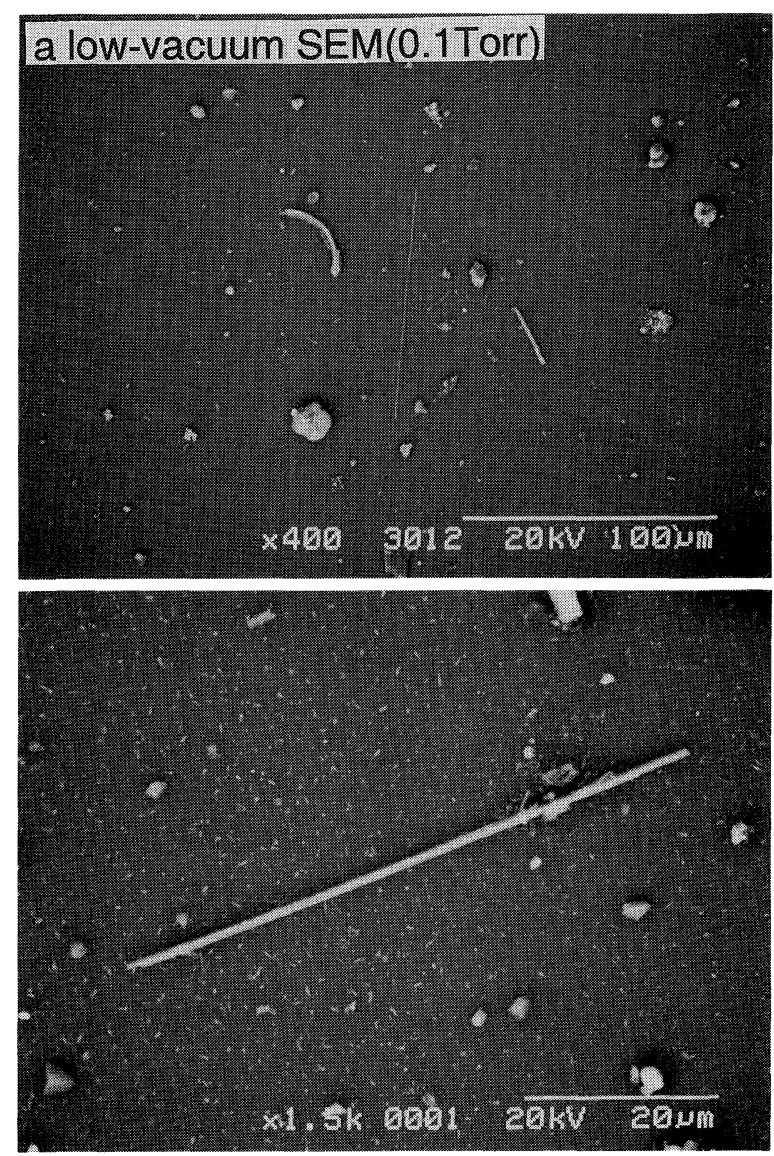

Fig. 12. Airborne amosite fibers observed by a low-vacuum SEM (0.1 Torr).

\section{Discussion}

PCM has been used as a monitoring tool for airborne asbestos fibers in working environments in many industrialized countries, according to legal regulations ${ }^{1,2)}$. It is obvious that the PCM method has many advantages and has played an important role in asbestos monitoring, not only for working environments but also in various other kinds of environments ${ }^{3,11)}$. However, as is well known, the PCM method has some limitations. It does not identify asbestos fibers alone, and may therefore give erroneously high results by including other fibers, such as gypsum, rock wool, whiskers, or cellulose, from factories or buildings where these asbestos substitutes are mixed with asbestos. It can not visualize thin fibers with 
diameters less than about $0.4 \mu \mathrm{m}$ because of limited resolution ${ }^{12)}$. These small fibers are believed to have major significance in carcinogenicity.

Since the end of the 1970s, several researchers have suggested that fiber size is an important factor of carcinogenicity. Stanton, et al. $(1978,1981)^{4,13)}$ and Pott $(1978,1980)^{5,14)}$, based on the animal experiments of mineral fibers, presented the idea that carcinogenicity of fibers increases as fiber length increases and diameter decreases: in particular, that fibers thinner than $0.25 \mu \mathrm{m}$ and longer than $8 \mu \mathrm{m}$ show the strongest carcinogenicity. The PCM method cannot detect these thin fibers, so the most dangerous fibers are not monitored by the PCM method. By contrast, TEM can resolve even the finest fibers that may be present and it can specifically identify such fibers. At present, TEM is a satisfactory method for monitoring and measuring these fine fibers. Actually, the Asbestos Hazard Emergency Response Act (AHERA) was enacted in U.S.A. in 1986, mandating the Environmental Protection Agency (EPA) to take certain actions to control asbestos hazards in schools. According to the act, EPA issued "Final Rule and Notice" ${ }^{\text {"7) }}$ mandating TEM measurement as a method to confirm clearance after asbestos-abatement operations.

Since the carcinogenicity of asbestos was widely confirmed, industries which had used asbestos fibers for their products gradually stopped or decreased their use of asbestos, and instead began to use asbestos substitutes in their products, such as man-made mineral fibers, whiskers, and some other natural mineral fibers. By these industrial changes, airborne fibers in working environments sometimes consist of other fibers as well as asbestos. Moreover, according to biological studies of mineral fibers by many researchers, it has also been revealed that the carcinogenicity of mineral fibers may depend upon not only on fiber size, but also on fiber types. For example, it was summarized by Doll $(1989)^{15)}$ that the carcinogenicity of crocidolite is most strong within asbestos, and amosite follows it, and chrysotile is lowest. On the contrary, chrysotile showed higher incidence of mesothelioma than amosite and crocidolite in animal experiments using rats ${ }^{16)}$, while erionite fibers, a zeolite mineral, showed stronger carcinogenicity than crocidolite, amosite, or chrysotile in an experiment using mice ${ }^{17}$. The author's group found an asbestos size whisker (rutile whisker) which did not show any carcinogenicity in the examination by intraperitoneal injection in rats ${ }^{18}$. Thus, it is important to know which fibers have strong carcinogenicity and which fibers are safer. This suggests the needs for measurement of airborne fibers for each fiber type will increase in the future.

Until now, many measurements by SEM and/or TEM have been undertaken in many environments ${ }^{5,19)}$, but these EM measurements were done independently from PCM measurement or using different filter samples than those used for PCM measurement. Given these conditions, the authors developed new sample preparation methods for OM, DS/PLM, SEM, and TEM measurements of mineral fi- 
bers on the same filter sample with collected airborne dust. The technique of lowtemperature ashing of a membrane filter attached to a glass slide was first developed for these preparation methods. This technique is simple but very important for these preparation methods. As shown in Figure 1, most sample preparation methods developed in this study were accomplished using this low-temperature ashing. These newly-developed sample preparation methods, as well as the traditional PCM method, were combined to develop a total sample preparation method in this paper.

When the analyst intends to measure the fibers on a filter by EM in addition to PCM measurement, the air sampling work load decreases because one filter sample can be used for all these measurements, and he can directly compare the results obtained by these different measurements on the same filter area.

Comparing the photographs taken by the PCM and OM method in Figures 6 and 7 , we can see the OM photograph shows sharper images and better contrast than that of PCM. Actually, the fiber concentration data obtained by the OM method was higher than that of the traditional PCM method (Table 1). The results by the OM method (Table 1) are equivalent to the data calculated for fibers larger than $0.3 \mu \mathrm{m}$ in diameter in Table 2, indicating that the OM method can observe fibers larger than $0.3 \mu \mathrm{m}$ in diameter, while the PCM method, as mentioned previously, only detects fibers larger than $0.4-0.5 \mu \mathrm{m}$ in diameter. Thus, counting error due to each analyst would become smaller in the OM method than in PCM. It is concluded that the OM method employing low-temperature ashing is suitable for fiber counting and can produce precise data. Using low-temperature ashing, DS/PLM measurement for airborne mineral fibers was also tested and became possible in this study.

The TEM photographs in Figures 8 and 9 taken by the TEM(1) method show a very wide field in a single picture, almost equivalent to that of PCM or OM, because it is obtained at a very low magnification of $200 \mathrm{x}$. Furthermore, it shows far clearer images of chrysotile fibers than those obtained by PCM or OM. We confirmed that the TEM(1) method can visualize almost all fibers down to 0.02 $\mu \mathrm{m}$ in diameter even at a low magnification such as 200 or $400 \mathrm{x}$ and provide us with a satisfactory data for size distributions of fibers. The TEM methods by $\mathrm{NIOSH}^{1)}, \mathrm{EPA}^{7)}$ and $\mathrm{ISO}^{8)}$ employ a direct-transfer procedure without using PVA solution. The procedure seems to be easier than our direct-transfer procedure of TEM(1), which uses PVA solution. However, as shown in this paper, the TEM image by TEM(1) is clear and can show a very fine fibers by observation at low magnifications. Therefore, the analyst can select and employ the best TEM method among these methods according to his purpose. Both parallel and serial preparation methods will be useful for the measurement of various fibers in airborne dust as well as in raw materials using comprehensive instruments such as PCM, OM, DS/PLM, SEM and TEM. 
This total method can be used not only for air samples but also for various types of samples such as liquid and biological tissue samples. In the case of human lung tissue, ferruginous bodies are mainly observed and counted by OM. Whether the core fibers are asbestos fibers is confirmed by analytical TEM for the same filter samples. In analysis by the analytical TEM, the types, sizes, and number of naked fibers are also measured, in the so-called "tissue burden study". One of the authors reported data obtained by this method for insulation workers in North America showing the number of fibers in the lung and other organs and confirmed the translocation of inhaled fibers from the lung to other organs ${ }^{20)}$. Asbestos and other fibers in liquid samples such as tap water, beer, mineral water, wine, waste water, etc., have also been analyzed by the authors using this total method.

In conclusion, we can obtain comprehensive data, including number, sizes, and types of fibers, for one filter sample using various measurements such as PCM, OM, DS/PLM, SEM, and TEM, by applying this total sample preparation method. The comprehensive data can contribute to the study of adverse health effects of asbestos and other fibers, and to an understanding of actual dose-response relationships.

\section{ACKNOWLEDGEMENTS}

The authors express their sincere thanks to Mr. M. Tomita of NICHIAS Co. Japan for his cooperation in sampling the airborne asbestos examined in this study.

\section{RERERENCES}

1) NIOSH Method 7400: Fibers. In: NIOSH Manual of Analytical Methods, 3rd ed. Cincinnati (OH); U.S. Department of Health, Education and Welfare 1989.

2) JAWE. Guidebook for Working Environment Measurement; I. Mineral dust and asbestos. Japan Association for Working Environment Measurement (JAWE), Tokyo 1990: 158 (in Japanese).

3) Environmental Agency of Japan. Manual for controlling asbestos emission. Examination Committee of Counter-Asbestos Pollution Sources, Gyosei, Tokyo 1985: (in Japanese).

4) Stanton MF, Layard M, Tegeris A, Miller E, May M, Morgan F, Smith A. Relation of particle dimension to carcinogenicity in amphibole asbestoses and other fibrous minerals. JNCI 1981;67: 965-75.

5) Pott F. Animal experiments on biological effects of mineral fibers. In: Wagner JC, ed. Biological Effects of Mineral Fibres, IARC Sci. Publ. No. 30, Lyon 1980: 261-72.

6) NIOSH Method7402: Asbestos Fibers. In: NIOSH Manual of Analytical Methods, 3rd ed. Cincinnati (OH); U.S. Department of Health, Education and Welfare 1989.

7) US EPA. Asbestos-Containing Materials in Schools. Final Rule and Notice, Federal Register 1987; 52: No. 210, 41826-905.

8) ISO 10312. Ambient Air-Determination of asbestos fibres-Direct-transfer transmission electron microscopy procedure 1993 (E).

9) ISO 13794. Ambient Air-Determination of asbestos fibres-Indirect-transfer transmission electron microscopy procedure $1993(\mathrm{E})$. 
10) McCrone WC. Asbestos Identification. McCrone Res. Inst., Chicago, IL, 1987.

11) Kohyama N. Airborne asbestos levels in non-occupational environments in Japan. In: Bignon J, Peto J, Saracci R, eds, Non-occupational Exposure to Mineral Fibres. IARC Sci Pub No. 90, International Agency for Research on Cancer, Lyon 1989; 262-75.

12) Kohyama N. Asbestos levels in the general environment and in human tissues. In: Gibbs GW, Dunningan J, Kido M, Higashi T, eds. Health Risks from Exposure to Mineral Fibres: An International Perspective. Captus Univ. Pub, Canada 1993; 98-109.

13) Stanton MF, Layard M. The carcinogenicity of fibrous materials. In: Gravatt CC, LaFleur PD, Heinrichs KFJ, eds. Workshop on Asbestos: Definitions and Measurement Methods. National Bureau of Standards Special Publication No. 506, Washington,D.C. 1978; 143-51.

14) Pott F. Some aspects on the dosimetry of the carcinogenic potency of asbestos and other fibrous dust. Staub-Reinhalt Luft 1978; 38: 486-90.

15) Doll R. Mineral fibres in the non-occupational environment: Concluding remarks. In: Bignon J, Peto J, Saracci R, eds, Non-occupational Exposure to Mineral Fibres. IARC Sci Pub No. 90, International Agency for Research on Cancer, Lyon 1989; 511-8.

16) Pott F, Schlipkoter HW, Ziem U, Spurny K, Huth F. New results from implantation experiments with mineral fibres. In: Biological Effects of Man-Made Mineral Fibres, Proceedings of a WHO/ IARC Conference in Copenhagen, 20-2 April 1982, 1984; Vol. 2: 286-302.

17) Suzuki Y, Kohyama N. Malignant mesothelioma induced by asbestos and zeolite in the mouse peritoneal cavity. Envir Res 1984; 35:277-92.

18) Fukuda K, Kohyama N. Carcinogenicity of some fibrous minerals examined by intraperitoneal injections in rats. J Occup Health 1995; 37: Supplement p.527 (in Japanese).

19) Sebastien P, Billon MA, Dufour G, Gaudichet A, Bonnaud G, Bignon J. Levels of asbestos air pollution in some environmental situations. Ann N Y Acad Sci 1979; 330:401-15.

20) Kohyama N, Suzuki Y. Analysis of asbestos fibers in lung parenchyma, pleural plaques, and mesothelioma tissues of North American insulation workers. Ann N Y Acad Sci 1991; 643: 27 52. 